\title{
Prediction of tidal currents using Bayesian machine learning
}

\author{
Dripta Sarkar ${ }^{1}$, Michael Osborne, Thomas Adcock \\ Department of Engineering Science, University of Oxford, Oxford - OX13PJ, United \\ Kingdom.
}

\begin{abstract}
We propose the use of machine learning techniques in the Bayesian framework for the prediction of tidal currents. Computer algorithms based on the classical harmonic analysis approach have been used for several decades in tidal predictions, however the method has several limitations in terms of handling of noise, expressing uncertainty, capturing non-sinusoidal, non-harmonic variations. There is a need for principled approaches which can handle uncertainty and accommodate noise in the data. In this work, we use Gaussian processes, a Bayesian non-parametric machine learning technique, to predict tidal currents. The probabilistic and non-parametric nature of the approach enables it to represent uncertainties in modelling and deal with complexities of the problem. The method makes use of kernel functions to capture structures in the data. The overall objective is to take advantage of the recent progress in machine learning to construct a robust algorithm. Using several sets of field data, we show that the machine learning approach can achieve better results than the traditional approaches.
\end{abstract}

Keywords: prediction, tidal currents, machine learning, Gaussian process

\footnotetext{
${ }^{1}$ Corresponding Author: dripta.academic@gmail.com
} 


\section{Introduction}

Tidal waves are produced by changes in the gravitational forces of the sun and the moon. Prediction of tidal currents are necessitated by practical requirements like navigation, protection from flooding, coastal management to recent

5 nomenon began with Newton pioneering the gravitational theory and then later Laplace deriving the expression for the tidal potential. There have been many advances in methodologies for tidal analysis since then. The most widely used method is that of the harmonic analysis (HA), where the observed tidal varifrequencies, with the amplitudes and phases determined using the least-squares fitting procedure. Computer codes based on HA have been used for decades for the prediction of tidal heights (1-D) and currents (2-D). Over the years various advances have been made to HA approach [see e.g. 1, 2, 3] ). Other techniques include tidal spectroscopy [4], and response method for unified tide and surge prediction [5], however they have not been widely adopted. HA has been extensively used in the analysis of stationary tidal (height) records, providing insights into the tidal dynamics. However, there are several shortcomings of this methodology. One of the challenging tasks in HA is the selection of tidal constituents, which if inaccurate can lead to overfitting of data or numerical issues 6]. Appropriate modelling of noise is another issue. In tidal analysis, signals which do not contribute to the tidal variations are classified as 'noise. In reality, there can be cases where the non-tidal signal is much stronger than the tidal e.g. the occurrence of a stormy event, and many of such effects are non-harmonic. the technique is not suitable for application to non-stationary data 6$]$. HA is also incapable of modelling the spatial variability of tides - this is not a big issue in modelling tidal heights which changes slowly in space, however tidal currents can vary sharply within short distances due to changes in bathymetry

and topography. As tides move into shallow waters, they are distorted resulting 
in overtides (higher harmonics of principal constituents) and compound tides (interaction between different constituents). Such interactions can lead to asymmetry in the flood and ebb magnitudes of the current, depending on the phase relationship [7]. In HA, nonlinear characteristics are incorporated with the inclusion of shallow water constituents, some of which may need to be inferred, and such operations are often difficult. Even more complexities can result near headlands [8], where complex flow structures can result in additional frequencies, which are not necessarily sinusoidal. In relation to the uncertainty estimation, HA generates confidence intervals for the current ellipse parameters (which are often large [3]). However, in a lot of practical applications it is more useful to generate confidence interval estimates directly in the time domain.

In this work, we present a novel approach to predict tidal currents using probabilistic machine learning techniques in the Bayesian framework, which provide principled approaches for dealing with uncertainty, and can tackle the challenges

45 of real world data $[9]$. Uncertainty could be introduced in many forms - ranging from measurement noise to uncertainty in the parameters of the model, and the mathematics of probability enables expressing the uncertainties [10]. Bayesian modelling approaches have been widely used in different disciplines e.g. geostatistics 11], meteorology [12], economics [13], spatial statistics [14], machine learning [15]. Gaussian process (GP), a Bayesian non-parametric approach, have been shown to be well-suited in solving a variety of time-series modelling problems [9] and in this work we pursue this methodology to model tidal current data. We introduce the application of Bayesian machine learning to the tidal current prediction problem which can address some of the shortcomings associated with traditional techniques -

- modelling nonlinear interactions not captured in the HA especially at locations of fast tidal currents

- accommodating uncertainties of all forms e.g. noise is included directly in the mathematical formulation

- modelling non-harmonic variations in the short-term resulting from mete- 
orological effects, barotropic to baroclinic conversion.

- generating confidence intervals directly in the time-domain.

The method can be used for the prediction from any generic tidal current timeseries data. We show that the machine learning approach can produce better predictions than the HA even in cases where the latter is considered to be good (achieve good accuracy). An initial report on the novel machine learning approach to tidal currents was made in [16] where analysis was performed on tidal current data from a numerical model. In this work we provide a detailed description of the methodology with extensive discussions and analysis with real world datasets as well as present new approaches to model short and strongly contaminated datasets.

A brief overview of tides and HA is provided in the next section, followed by an introduction to GP regression. We then analyze long tidal current time series data using maximum a-posteriori and short tidal time-series data using Monte Carlo Markov chain techniques, and compare the results with classical HA approach. The work potentially opens up application of machine learning to other problems in tidal analysis which are otherwise not possible using traditional techniques.

\section{Tides and Harmonic analysis}

Based on potential field theory, forces due to the sun and the moon produce hundreds of tidal constituents with distinct frequencies. Nonlinear interaction of the astronomical tidal components produces secondary tides known as overtides (higher harmonics) or compound tides (interaction between various tidal constituents). Let us consider a time series: $y(t), t=t_{1}, t_{2}, \ldots, t_{M}$, where the observation times are regularly spaced at an interval $\Delta t$. The model equation with $N$ constituents can be expressed as

$$
y(t)=\sum_{k=1}^{N}\left(a_{k}^{+} e^{i \omega_{k}\left(t-t_{0}\right)+i V_{k}}+a_{k}^{-} e^{-i \omega_{k}\left(t-t_{0}\right)-i V_{k}}\right)+c_{0}+c_{1}\left(t-t_{0}\right)
$$


where $c_{0}$ is some offset and $c_{1}$ indicate the trend, while the term inside the summation indicate the variation of the constituents with $a_{k}^{+}$and $a_{k}^{-}$and being the unknown complex amplitudes, $\omega_{k}$ the angular frequency and $V_{k}$ is some astronomical argument. Note, $y(t)$ is real if modelling tidal heights, while for tidal currents it is a complex variable: $y(t)=u(t)+i v(t)$, and as such the equation in (1) can be expressed in matrix form as:

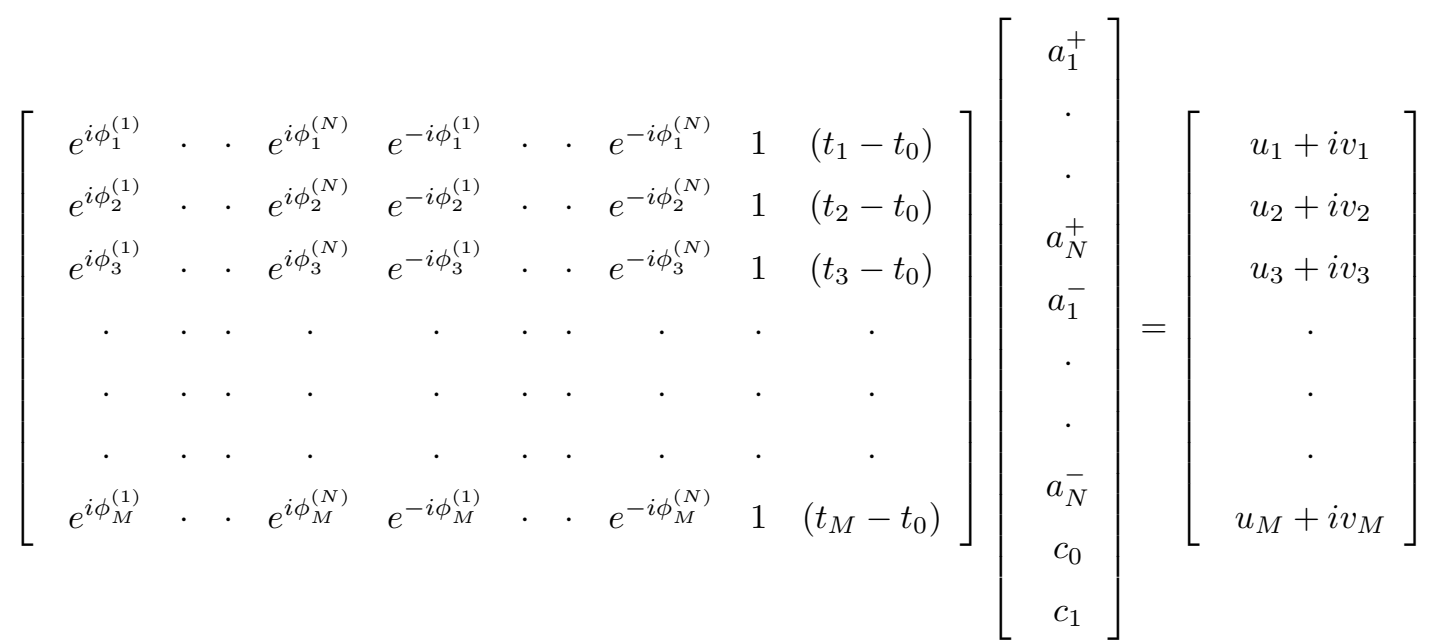

where $\phi_{m}^{(k)}=\omega_{k}\left(t_{m}-t_{0}\right)+V_{k}\left(t_{m}\right)$. The solutions are determined by minimizing some function of the residual $(\boldsymbol{T a}-\boldsymbol{y})$, where $\boldsymbol{y}=\left[y\left(t_{1}\right), y\left(t_{2}\right), \ldots, y\left(t_{m}\right)\right]^{\prime}$, $\boldsymbol{a}=\left[a_{1}^{+}, a_{2}^{+}, \ldots, a_{N}^{+}, a_{1}^{-}, a_{2}^{-}, \ldots, a_{N}^{-}, c_{0}, c_{1}\right]^{\prime}$ and $\boldsymbol{T}$ is a $M \times 2 N+2$ of linear and sinusoidal basis functions evaluated at the observation times. In case of the ordinary least squares (OLS) approach the objective function to be minimized can be expressed as $\|\boldsymbol{T} \boldsymbol{a}-\boldsymbol{y}\|^{2}$, and the solutions are determined as $\boldsymbol{a}=\left(\boldsymbol{T}^{*} \boldsymbol{T}\right)^{-1} \boldsymbol{T}^{*} \boldsymbol{y}$ where superscript $*$ indicates the conjugate transpose of the matrix. However, a shortcoming of the OLS method is its sensitivity to non-tidal variations, as it can over-fit such effects while trying to minimize the residual error [3]. The latest codes based on the HA uses the 'Iteratively Reweighted Least Squares' algorithm which reduces the influence of the non-tidal effects and the solution in this case is obtained as $\boldsymbol{a}=\left(\boldsymbol{T}^{*} \boldsymbol{W} \boldsymbol{T}\right)^{-1} \boldsymbol{T}^{*} \boldsymbol{W} \boldsymbol{y}$, where $\boldsymbol{W}$ is some weighting matrix which is determined iteratively (see [3] for details). The standard 


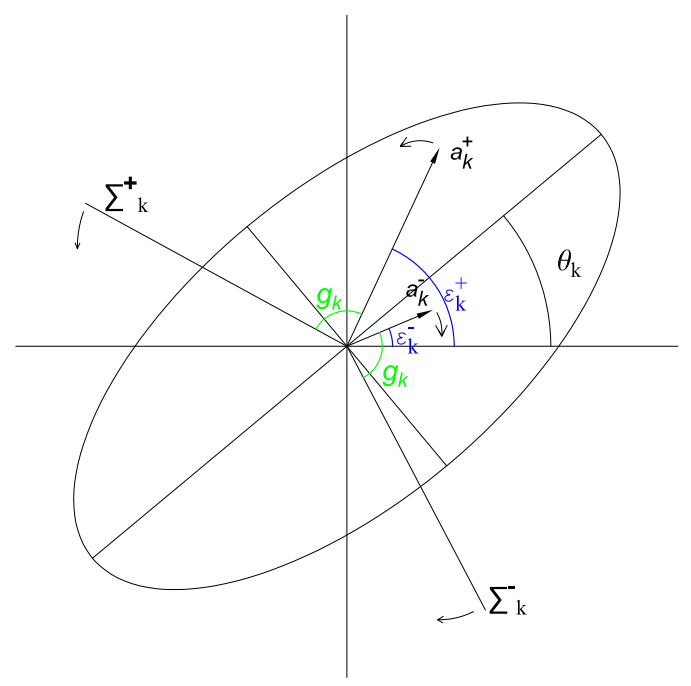

Figure 1: For a particular tidal constituent $k$, the rotating vectors with amplitude $a_{k}^{+}$and $a_{k}^{-}$are considered to be generated by two different fictitious stars $\sum_{k}^{+}$and $\sum_{k}^{-}$, rotating in the counterclockwise and clockwise direction respectively, at a speed same as that of the constituents. The constant phase angle by which the rotating vectors lead or lag behind their respective stars is known as the greenwich phase $g_{k}$.

parameters of the particular constituent $k$ are then computed as

$$
\begin{gathered}
L_{k}=\left|a_{k}^{+}\right|+\left|a_{k}^{-}\right|, \quad l_{k}=\left|a_{k}^{+}\right|-\left|a_{k}^{-}\right| \\
\theta_{k}=\left(\frac{\alpha_{k}^{+}+\alpha_{k}^{-}}{2}\right), \quad g_{k}=v_{k}-\left(\frac{\alpha_{k}^{+}-\alpha_{k}^{-}}{2}\right)
\end{gathered}
$$

where $L_{k}$ and $l_{k}$ are the semi-major and semi-minor axis of the ellipse respectively, $\alpha_{k}^{+}=\operatorname{ang}\left(a_{k}^{+}\right), \alpha_{k}^{-}=\operatorname{ang}\left(a_{k}^{-}\right), \theta_{k}$ is the angle of inclination of the northern semi-major axis counter-clockwise from due east, and $g_{k}$ is the Greenwich phase [see 17]. The parameters could be visualized as shown in figure 1.

\subsection{Selection of constituents}

There exists over 500 tidal constituents [see e.g. 2], considering all astronomical and shallow water constituents however, inclusion of all of them the HA demands almost 19 years of continuous data, which is hard to obtain in 

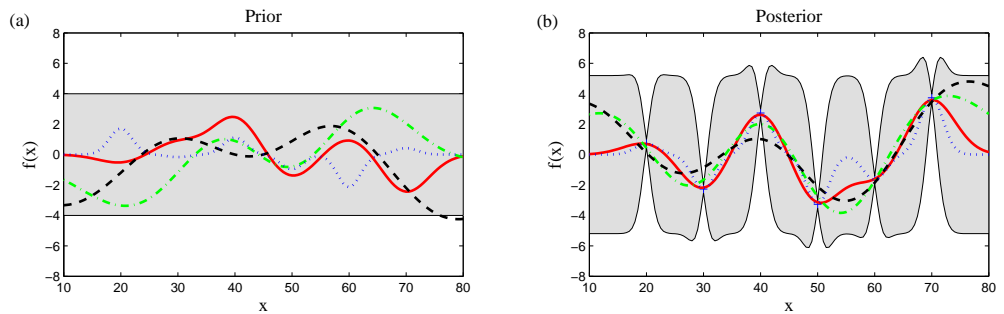

Figure 2: GPs describe a prior distribution in the space of functions. The prior distribution expresses our belief in problem understanding before seeing the data (figure a). Once inference is performed, the posterior distribution can be described. Essentially this is the distribution of functions which with some added noise passes through the given data points. There is reduced uncertainty at locations near the observations.

reality. In practice, the choice of constituents are made through the automated selection algorithm developed by [18], where constituents are selected from a basis of 45 astronomical and 24 of the most important shallow water constituents. The Rayleigh criterion [19] is used for constituent selection, whereby a time series of minimum length $T_{0}$ can distinguish tidal constituents with a frequency difference of $T_{0}^{-1}$. If two constituents do not satisfy the Rayleigh criterion, then the one with a larger amplitude in the equilibrium analysis is considered in the $\mathrm{HA}$, with the other constituent ignored. HA is not suitable for application to non-stationary processes unless the frequencies of the non-stationary processes, if harmonic with known frequencies, are included in the analysis [6].

\section{Gaussian Process Regression}

GPs represent a Bayesian non-parametric approach to modelling unknown functions, and have been widely used in solving a variety of regression problems [10, 15]. The modelling framework considers a prior distribution directly in the space of functions (see figure 21), with the properties of such functions governed by the mean and covariance function of the GP - similar to the description of a multivariate Gaussian distribution in terms of its mean and covariance matrix [20]. Consider a dataset with $n$ observations $\mathcal{D}=\left\{\left(x_{i}, y_{i}\right), i=1,2, . ., n\right\}$ and the objective is to make predictions for new inputs $\boldsymbol{x}_{*}$. The outputs $\boldsymbol{y}$ are generated 
by a latent function $f(x)$ with the addition of Gaussian white noise of constant variance $\left(\sigma_{n}^{2}\right)$

$$
y_{j}=f\left(x_{j}\right)+\varepsilon_{j}, \quad \varepsilon_{j} \sim \mathcal{N}\left(0, \sigma_{n}^{2}\right) .
$$

A prior distribution is considered on the latent functions such that for a given set of training points $\boldsymbol{x}=\left\{x_{1}, x_{2}, \ldots, x_{n}\right\}$, the corresponding vector of function evaluations is given by the $n$ dimensional Gaussian distribution $\boldsymbol{f} \sim \mathcal{N}\left(\boldsymbol{m}_{n}, \boldsymbol{K}_{n}\right)$, and the properties of the prior are dictated by the choice of the covariance function $\boldsymbol{K}_{n}(i, j)=\mathbb{E}\left[\left(f\left(x_{i}\right)-m\left(x_{i}\right)\right)\left(f\left(x_{j}\right)-m\left(x_{j}\right)\right)\right]$. Note, the symbol $\sim$ means 'distributed according to'. The specification of the prior is important as it fixes the properties of the function considered for inference [15]. The model likelihood function represents the difference between noisy measurements and the underlying noise-free function [15]

$$
p(\boldsymbol{y} \mid \boldsymbol{f})=\mathcal{N}\left(\boldsymbol{y} ; \boldsymbol{f}, \sigma_{n}^{2} \boldsymbol{I}\right),
$$

where $\mathbf{I}$ is the identity matrix. Employing Bayes theorem, the distribution of latent function values given the data points can be expressed as

$$
p(\boldsymbol{f} \mid \boldsymbol{y})=\frac{p(\boldsymbol{y} \mid \boldsymbol{f}) p(\boldsymbol{f})}{\int p(\boldsymbol{y} \mid \boldsymbol{f}) p(\boldsymbol{f}) \mathrm{d} \boldsymbol{f}} .
$$

Since in the GP framework we have a multi-dimensional Gaussian distribution for any finite number of input points, the outputs of the latent function at the training and test location can then be given by the distribution

$$
\left[\begin{array}{c}
\boldsymbol{f} \\
\boldsymbol{f}_{*}
\end{array}\right] \sim \mathcal{N}\left(\left[\begin{array}{c}
\mathbf{m}_{n} \\
\mathbf{m}_{*}
\end{array}\right], \quad\left[\begin{array}{cc}
\boldsymbol{K}_{n} & \boldsymbol{K}_{n *} \\
\boldsymbol{K}_{* n} & \boldsymbol{K}_{* *}
\end{array}\right] .\right)
$$

where $\boldsymbol{K}_{n}$ is the covariance matrix expressing the correlations between all the training points, $\boldsymbol{K}_{n *}$ is the vector of covariance between the training points and the test target, $\boldsymbol{K}_{* *}$ is the prior variance at the test locations plus noise $\left(\sigma_{n}^{2}\right)$ and $\boldsymbol{y}_{*}$ are the actual output values at the test locations. Using Gaussian identities, we then have the conditional distribution

$$
p\left(\boldsymbol{f}_{*} \mid \boldsymbol{f}\right)=\mathcal{N}\left(\boldsymbol{m}_{*}+\boldsymbol{K}_{* n} \boldsymbol{K}_{n}^{-1}(\boldsymbol{f}-\boldsymbol{m}), \boldsymbol{K}_{* *}-\boldsymbol{K}_{* n} \boldsymbol{K}_{n}^{-1} \boldsymbol{K}_{n *}\right)
$$




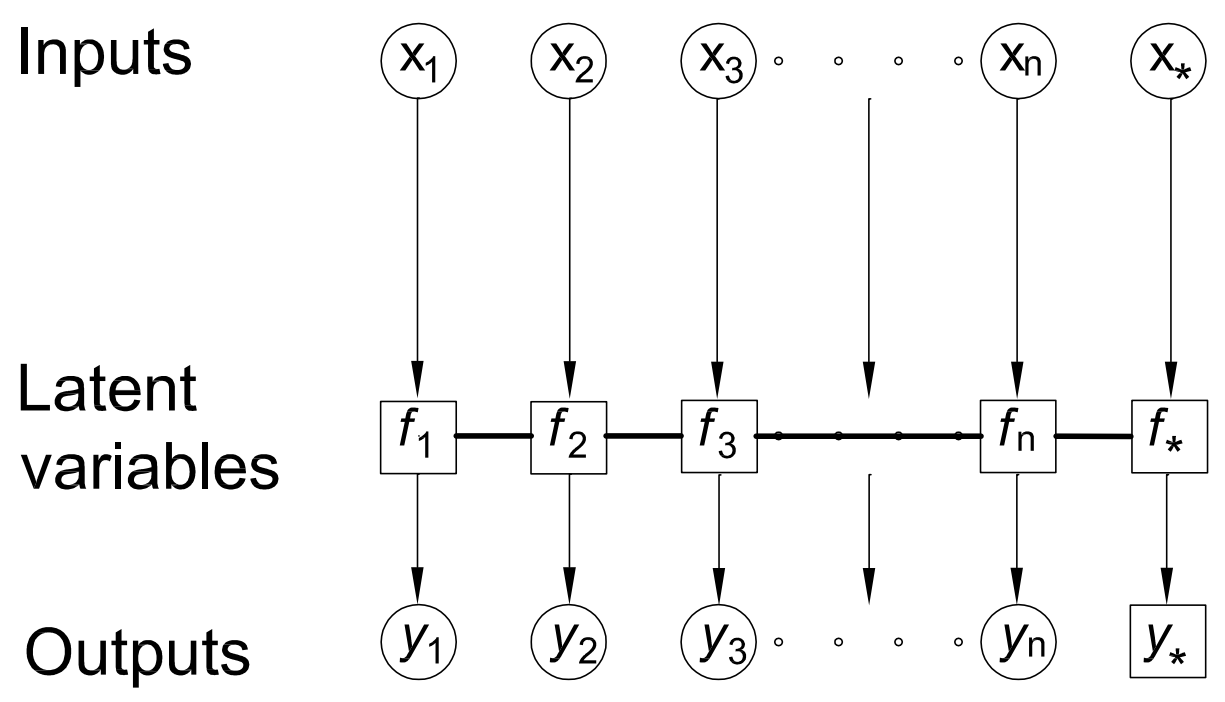

Figure 3: Structure of Bayesian Gaussian process model for regression. The bold horizontal line represents a fully connected set of nodes. The variables within square boxes are unknown quantities. The variable $y_{*}$ is the prediction at the unobserved input location $x_{*}$.

which is employed to determine the function values $\boldsymbol{f}_{*}$ at the unknown locations

$$
p\left(\boldsymbol{f}_{*} \mid \boldsymbol{y}\right)=\int p\left(\boldsymbol{f}_{*} \mid \boldsymbol{f}\right) p(\boldsymbol{f} \mid \boldsymbol{y}) \mathrm{d} \boldsymbol{f}
$$

Finally, the predictions of the output values $\boldsymbol{y}_{*}$ can be given by

$$
p\left(\mathbf{y}_{*} \mid \mathbf{y}\right)=\int p\left(\mathbf{y}_{*} \mid \mathbf{f}_{*}\right) p\left(\mathbf{f}_{*} \mid \mathbf{y}\right) \mathrm{d} \mathbf{f}_{*}=\mathcal{N}\left(\boldsymbol{\mu}_{*}, \boldsymbol{\sigma}_{*}^{2}\right)
$$

where

$$
\begin{aligned}
& \boldsymbol{\mu}_{*}=\mathbf{m}_{*}+\mathbf{K}_{* n}\left(\mathbf{K}_{n}+\sigma_{n}^{2} \mathbf{I}\right)^{-1}\left(\mathbf{y}-\mathbf{m}_{n}\right) \\
& \boldsymbol{\sigma}_{*}^{2}=\mathbf{K}_{* *}+\sigma_{n}^{2} \mathbf{I}-\mathbf{K}_{* n}\left(\mathbf{K}_{n}+\sigma_{n}^{2} \mathbf{I}\right)^{-1} \mathbf{K}_{n *},
\end{aligned}
$$

with $\mathbf{K}_{n *}$ being the matrix of covariance between the training points and test locations and $\mathbf{K}_{n *}=\mathbf{K}_{* n}^{T}$. The mean function in the present application is considered to be some constant plus a linear trend with respect to time. The trend term can be ignored if desired. Overall the structure of the Bayesian GP can be graphically summarized as in figure 3 . 


\subsection{Kernel function}

Various forms of the kernel function are known and they can be combined in numerous way (addition, multiplication), depending upon the problem, to model complex data, however the resultant matrix has to be positive semidefinite [15]. Note, in the machine learning literature as well as in this paper, the terms kernel function and covariance function are interchangeably used to denote the same function. The covariance matrix is generated by the kernel function. Designing an appropriate kernel function is an important aspect of a machine learning problem. The exponentiated quadratic is one kernel function which is ubiquitously used in solving a wide variety of problems

$$
\boldsymbol{K}_{n}(i, j)=\sigma_{f, q}^{2} \exp \left(\frac{\left|x_{i}-x_{j}\right|^{2}}{l_{q}^{2}}\right) .
$$

Another popular kernel function is the Matérn kernel function [see e.g. 15], which is more appropriate for modelling rougher (non-smooth) variations. For the particular problem of tidal current prediction, given the harmonic nature of the variations, a good choice of a kernel function would be a periodic kernel function

$$
\boldsymbol{K}_{n}(i, j)=\sigma_{f, q}^{2} \exp \left(\frac{-2}{l_{q}^{2}} \sin ^{2}\left(\frac{\pi\left|x_{i}-x_{j}\right|}{p_{q}}\right)\right)
$$

and given the multiple number of distinct periodic tidal constituents, addition of finite number of periodic kernel functions could be appropriate for the present application.

The variation of a periodic kernel function with same frequency and signal variance but different length-scales is illustrated in figure 4. One of the key strengths of the periodic kernel function is its potential in capturing nonsinusoidal variations.

The posterior distribution $p\left(\mathbf{y}^{*} \mid \theta, \mathbf{y}\right)$ is a function of unknown hyperparameters $\theta$ which needs to be marginalized (integrating out the uncertainty) as

$$
p\left(\mathbf{y}_{*} \mid \mathbf{y}\right)=\int p\left(\mathbf{y}_{*} \mid \boldsymbol{\theta}, \mathbf{y}\right) p(\boldsymbol{\theta} \mid \mathbf{y}) \mathrm{d} \boldsymbol{\theta},
$$



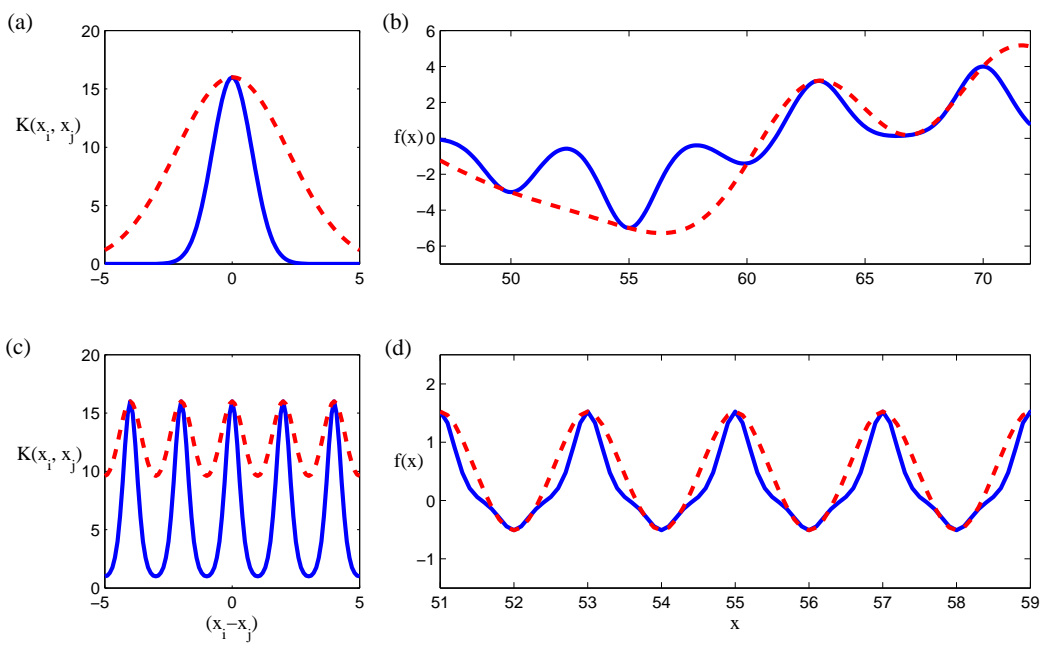

Figure 4: Figure (a) shows an exponentiated quadratic kernel function with two different lengthscales, while figure (b) shows example draws from a GP prior with those length scales. The red line corresponds to a longer length-scale than the blue line. Figure (c) and (d) shows the same but for a periodic kernel function.

where $p(\boldsymbol{\theta} \mid \mathbf{y})$ is the posterior distribution of the hyperparameters. Using the Bayes theorem to $p(\boldsymbol{\theta} \mid \mathbf{y})$, the above equation can be expressed as

$$
p\left(\mathbf{y}_{*} \mid \mathbf{y}\right)=\frac{\int p\left(\mathbf{y}_{*} \mid \theta, \mathbf{y}\right) p(\mathbf{y} \mid \boldsymbol{\theta}) p(\boldsymbol{\theta}) \mathrm{d} \boldsymbol{\theta}}{\int p(\mathbf{y} \mid \boldsymbol{\theta}) p(\boldsymbol{\theta}) \mathrm{d} \boldsymbol{\theta}}
$$

where $p(\mathbf{y} \mid \boldsymbol{\theta})$ is known as the likelihood and $p(\boldsymbol{\theta})$ is the prior distribution on the unknown hyperparameters. One of the main challenges in the GP approach is finding a solution to the intractable integrals in (77) and (8). Two well known approaches of determining an approximate solution are the maximum likelihood estimation (MLE) also known as the type-II maximum likelihood, and the maximum a-posteriori (MAP) approach. Both these approach typically work well with large datasets where the likelihood function is typically highly peaked. The MLE approach approximates the likelihood function $p(\boldsymbol{y} \mid \boldsymbol{\theta})$ as a delta function at the location of the hyperparameters maximizing it: 


$$
\begin{aligned}
& \log p\left(\boldsymbol{y} \mid \theta_{\mathbf{M L E}}\right)=\underbrace{-\frac{1}{2}(\boldsymbol{y}-\boldsymbol{\mu})^{T}\left(\mathbf{K}+\sigma_{n}^{2} \mathbf{I}_{n}\right)^{-1}(\boldsymbol{y}-\boldsymbol{\mu})}_{\text {penalizes mismatch between data and prediction }} \overbrace{-\frac{1}{2}\left|\mathbf{K}+\sigma_{n}^{2} \mathbf{I}_{n}\right|}^{\text {penalizes model complexity }} \\
&-\frac{n}{2} \log (2 \pi),
\end{aligned}
$$

where $\boldsymbol{\mu}$ indicate the predictions at the training locations $\boldsymbol{x}$. In the MAP method, the hyperparameter posterior $p(\boldsymbol{\theta} \mid \mathbf{y})$ in equation (7) is considered to be a Dirac delta function at its maximum, with the optimization performed on:

$$
\begin{array}{r}
\log p\left(\boldsymbol{\theta}_{M A P} \mid \boldsymbol{y}\right)=-\frac{1}{2}(\boldsymbol{y}-\boldsymbol{\mu})^{T}\left(\mathbf{K}+\sigma_{n}^{2} \mathbf{I}_{n}\right)^{-1}(\boldsymbol{y}-\boldsymbol{\mu})-\frac{1}{2}\left|\mathbf{K}+\sigma_{n}^{2} \mathbf{I}_{n}\right| \\
-\frac{n}{2} \log (2 \pi)+\log p\left(\boldsymbol{\theta}_{M A P}\right)
\end{array}
$$

The prior $p\left(\boldsymbol{\theta}_{M A P}\right)$ could either be approximated with a flat distribution or a probability distribution could be assigned to it. The optimization is performed using the method of conjugate gradients. For large tidal current datasets, we adopt the MAP approach for determining the hyperparameters as it incorporates additional information through hyperparameter prior distributions $p\left(\boldsymbol{\theta}_{M A P}\right)$. For short time-series data, the approximation of hyperparameter posterior as a Dirac delta function can often become inappropriate. A Markov chain Monte Carlo (MCMC) in the framework of Hamltonian Monte Carlo can be used to determine a solution to (7) in such circumstances. The method simulates a fictitious Hamiltonian dynamical system to achieve better exploration of the posterior distribution of the hyperparameters [see 21].

\section{Multi-Output}

The formulation described until now does not account for the correlations between the output variables, which in the case of tidal current prediction are the horizontal velocities $u$ and $v$. In the GP framework, the problem of multiple output reduces to the specification of an appropriate covariance function, which 
while being positive semi-definite, captures the dependencies between the data points across all the outputs [22].

The covariance function for multiple output can be expressed as

$$
\boldsymbol{K}_{n}=\sum_{q=1}^{M} \boldsymbol{\Upsilon}_{q} \otimes \boldsymbol{K}_{q}
$$

where $M$ indicates the finite number of frequencies considered from astronomical considerations,

$$
\boldsymbol{K}_{q}(i, j)=\exp \left(\frac{-2}{l_{q}^{2}} \sin ^{2}\left(\frac{\pi\left|x_{i}-x_{j}\right|}{p_{q}}\right)\right)
$$

and the matrix $\boldsymbol{\Upsilon}_{q}$ is assumed to be of spherical parametrization kind [23, 24] with

$$
\boldsymbol{\Upsilon}_{q}=\operatorname{diag}\left(\boldsymbol{e}_{q}\right) \boldsymbol{S}_{q}^{T} \boldsymbol{S}_{q} \operatorname{diag}\left(\boldsymbol{e}_{q}\right)
$$

For example, where we have only two outputs, in (13), $e_{q}$ gives a description for the length scale of each output variable

$$
\operatorname{diag}\left(\boldsymbol{e}_{q}\right)=\left(\begin{array}{cc}
l_{u, q} & 0 \\
0 & l_{v, q}
\end{array}\right)
$$

and $\boldsymbol{S}$ is an upper triangular matrix, the $i$ th column of which is associated with particular spherical coordinates of points of $\Re^{i}$ :

$$
\boldsymbol{S}_{q}=\left(\begin{array}{cc}
1 & \cos \theta_{q} \\
0 & \sin \theta_{q}
\end{array}\right)
$$

and the final form of the coregionalization matrix is given by

$$
\mathbf{\Upsilon}_{q}=\left(\begin{array}{cc}
l_{u, q}^{2} & l_{u, q} l_{v, q} \cos \theta_{q} \\
l_{u, q} l_{v, q} \cos \theta_{q} & l_{v, q}^{2}
\end{array}\right) \text {. }
$$

\section{Analysis}

The data analyzed in this work are from public datasets available through National Oceanic and Atmospheric Administration (NOAA), USA (www.noaa.gov). 

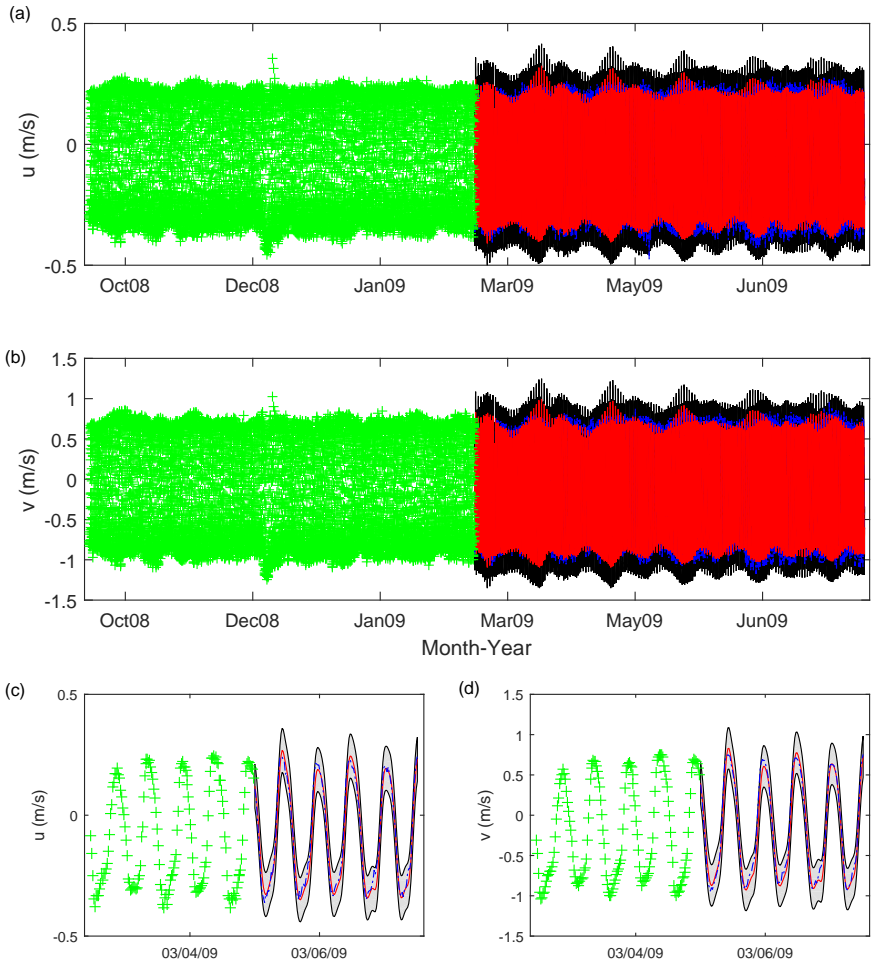

Figure 5: The figure shows the variations of the horizontal velocities $u$ and $v$ with time at Sunshine Sky Bridge. Five months of data is used to predict the tidal current variations for the next five months. The green ' + ' markers indicate the training points, the red solid line indicates the mean of the predictions, the black solid line containing the region in grey indicates the $95 \%$ confidence interval and the blue dotted line indicates the actual values. The figures (a)and (b) provide a good macroscopic view of the tidal prediction, however the details are obscured due to the vast amount of information it contains. Figures (c) and (d) shows a zoomed portion of figures (a) and (b) respectively, including the transition from training to prediction zone in both cases. The last two figures clearly shows the predicted mean (red) and confidence interval (grey), along with the periodic nature of the variations.

The classical HA is performed using the state-of-the-art Unified Tidal analysis toolbox (UTide) developed by [25] using the 'Iteratively Reweighted Least 145 Squared' (IRLS) algorithm [3] which performs a weighted statistical fitting such 

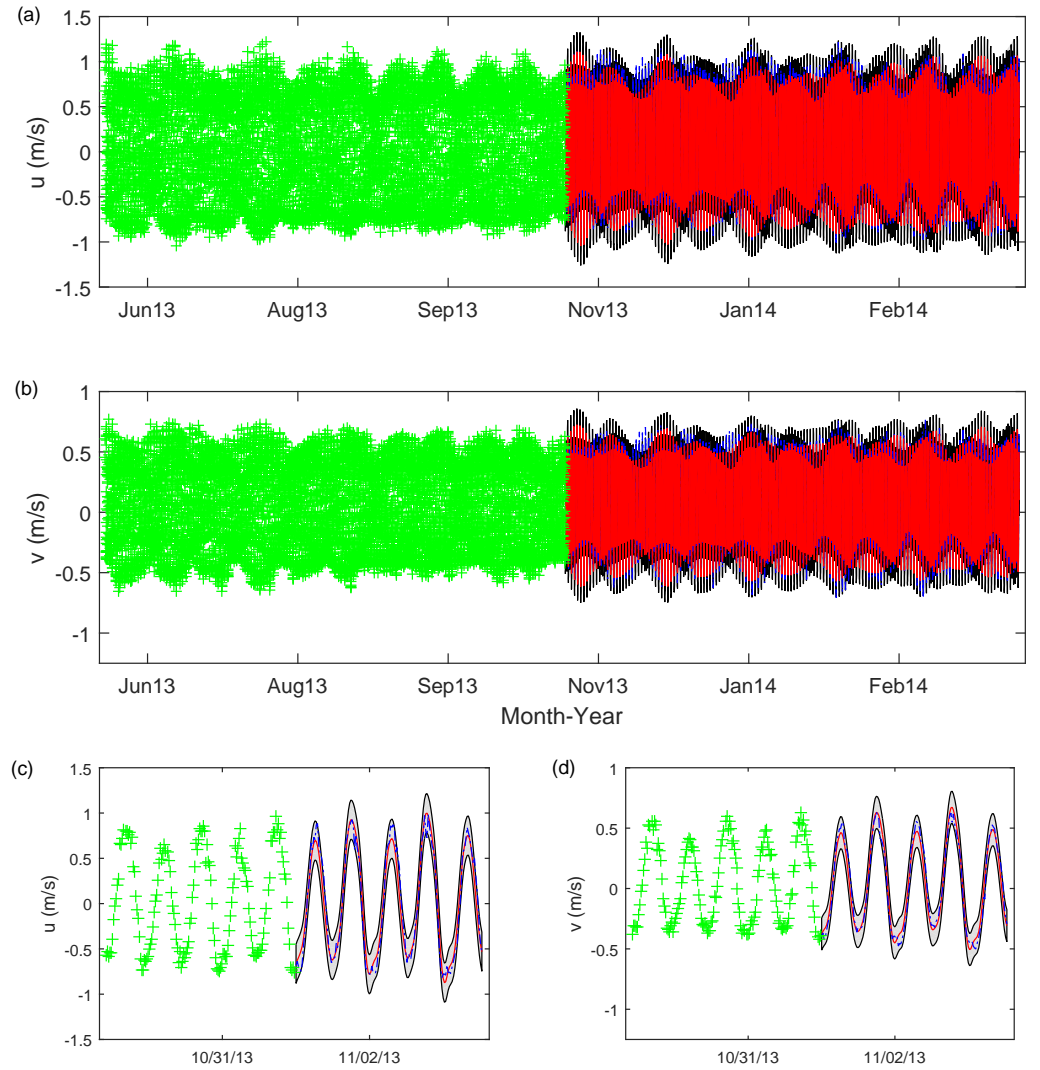

Figure 6: The figure shows the variations of the horizontal velocities $u$ and $v$ with time at Martinez-AMORCO Pier. Five months of data is used to predict the tidal current variations for the next five months. The details are the same as the previous figure.

that the effect of the outliers are reduced. An important point to note is that the GP is a non-parametric method, and the hyperparameters - which are unknown - are different from parameters in parametric models (HA). The hyperparameters (e.g. length-scale, variance, periodicity) govern the properties of functions chosen from the GP. They do not necessarily have physical interpretations, although the periodicity hyperparameters have some correspondence to the har- 


\begin{tabular}{lrr}
\hline Location & Latitude & Longitude \\
\hline Southampton Shoal Channel & $37^{\circ} 54.975^{\prime} \mathrm{N}$ & $122^{\circ} 25.340^{\prime} \mathrm{W}$ \\
Old Port Tampa & $27^{\circ} 51.772^{\prime} \mathrm{N}$ & $82^{\circ} 33.224^{\prime} \mathrm{W}$ \\
Sunshine Sky Bridge & $27^{\circ} 37.500^{\prime} \mathrm{N}$ & $82^{\circ} 39.300^{\prime} \mathrm{W}$ \\
Martinez-AMORCO Pier & $38^{\circ} 2.078^{\prime} \mathrm{N}$ & $122^{\circ} 7.515^{\prime} \mathrm{W}$ \\
\hline
\end{tabular}

Table 1: Location of the tidal observation sites for long term predictions. The current measurements at all the four locations are made at near surface.

monic nature of the tidal data. Computations for the two approaches (HA and GP) are performed with the same number of tidal constituents, chosen by the auto-selection algorithm developed by [18]. However note that the Rayleigh criterion decides the number of periodic kernel components of the GP; the form of the periodic kernel component enables the GP to capture more nonlinearities than HA. The frequencies of the periodic covariance functions in the GP formulation are specified to be the frequencies determined by the auto-selection procedure [18]. The locations of the longer datasets are presented in table 1 The data used in the analysis is sampled once every 24 minutes. Figures 5 (a) and (b) plots the variation of the horizontal velocities ( $u$ and $v$ ) versus time obtained using GP for one of the locations - Sunshine Sky Bridge (see Table11). The mean and confidence margins of the predictions agree well with the data. Due to the vast amount of information contained in figure 5, the short-term variations and the details are not properly visible. Figures 5(c) and (d) presents a short section of the figures 5 (a) and (b) respectively, illustrating the details of the GP outputs and the velocity data.

Table 2 shows the root mean squared error (RMSE) of tidal current predictions using the GP and comparisons are made alongside with the HA approach. The first column shows the location, the second column shows the training duration, then the prediction/test duration followed by the errors in $u$ and $v$ velocities respectively. Four locations are considered and predictions are performed over 1 month, 2 months and 5 months for each of the cases. The auto-selection 
algorithm selects 35 tidal constituents for all the four cases presented in table 175 2. with a minimum threshold factor of one in the Rayleigh criterion. The GP approach achieves better accuracy than the HA in each of the cases. On an average, the GP approach reduces the RMSE by around 5 percent from the $\mathrm{HA}$, and in some cases doing better by as much as 10 percent. The observed fluctuations in the errors over different prediction horizons can be attributed to variations in the real-world data arising from non-tidal contributions, as well as from low frequency tidal components which are not separated due to the lack of training data. Non-tidal effects can originate from sub-tidal variations, storm surges, etc. The purpose of the comparison is to show that the errors are comparable over different prediction horizons.

185 The training of the GP for the above cases is performed using an optimization algorithm, and the computational time depends on the number of function evaluations. On an average, the computational costs involved in using the GP are in the order of hours, while the HA takes seconds to evaluate. The HA is a parametric approach and is quasi-instantaneous; in contrast the Bayesian GP is non-parametric with the number of parameters growing with data, and as such takes more time for evaluation. This shortcoming can be addressed by using scalable approaches to GP inference [26, 27], and the results can be achieved in times comparable to that of HA. 3 for the locations). Some of the locations record quite strong tidal currents (Avatanak and Derbin Strait), which could make them suitable for tidal energy projects. Approximately half of the available data is used for training, while the rest for predictions. The auto-selection algorithm selects 17 tidal constituents for all these cases. Table 4 shows the RMSE of the predictions from the HA and GP approach for the short datasets. In this case again, the GP based approach again outperforms the HA.

Some of the cases in table 4 have much larger RMSE than those in table 2 A general increase in error can be ascribed to the shorter length of input data in 


\begin{tabular}{|c|c|c|c|c|c|c|}
\hline \multirow[b]{2}{*}{ Location } & \multirow[b]{2}{*}{ Training Duration } & \multirow[b]{2}{*}{ Prediction } & \multicolumn{2}{|c|}{$u$} & \multicolumn{2}{|c|}{$v$} \\
\hline & & & HA & GP & HA & GP \\
\hline Southampton & $25 / 03 / 13-24 / 08 / 13$ & 1 month & 0.0695 & 0.0668 & 0.1137 & 0.1083 \\
\hline \multirow[t]{2}{*}{ Shoal Channel } & & 2 months & 0.0718 & 0.0687 & 0.1104 & 0.1050 \\
\hline & & 5 months & 0.0738 & 0.0720 & 0.1042 & 0.1008 \\
\hline \multirow[t]{3}{*}{ Old Port Tampa } & $15 / 01 / 13-14 / 06 / 13$ & 1 month & 0.0573 & 0.0532 & 0.1179 & 0.1092 \\
\hline & & 2 months & 0.0562 & 0.0523 & 0.1174 & 0.1090 \\
\hline & & 5 months & 0.0634 & 0.0586 & 0.1310 & 0.1158 \\
\hline Sunshine Sky & $29 / 04 / 14-28 / 09 / 14$ & 1 month & 0.1340 & 0.1247 & 0.0916 & 0.0836 \\
\hline \multirow[t]{2}{*}{ Bridge } & & 2 months & 0.1375 & 0.1337 & 0.0942 & 0.0904 \\
\hline & & 5 months & 0.1252 & 0.1236 & 0.0841 & 0.0825 \\
\hline \multirow[t]{3}{*}{ Martinez } & $29 / 04 / 14-28 / 09 / 14$ & 1 month & 0.1322 & 0.1250 & 0.0787 & 0.0756 \\
\hline & & 2 months & 0.1297 & 0.1239 & 0.0773 & 0.0735 \\
\hline & & 5 months & 0.1208 & 0.1180 & 0.0743 & 0.0713 \\
\hline
\end{tabular}

Table 2: Comparison of the RMSE (in $\mathrm{m} / \mathrm{s}$ ) of the prediction using the HA and GP algorithm. The first column (left-most) indicate the locations, the second column shows the 5 month training period for the algorithms, while the third column present the prediction durations starting one day after the end of the training algorithm. All data for training and testing have been obtained from NOAA.

table 4, and fewer tidal constituents are selected in the latter. However, the first two cases in table 4 (Derbin and Avatanak Strait) have the largest magnitudes of tidal currents, and produces the largest values of RMSEs, although their percentage errors, with respect to overall current magnitudes, are similar to others.

In many cases, we are interested in unified prediction of currents (tidal+nontidal). The non-tidal effects are often non-harmonic and can have energy com- 


\begin{tabular}{lrrr}
\hline Location & Latitude & Longitude & Approx. Depth (m) \\
\hline Derbin Strait & $54^{\circ} 5.035^{\prime} \mathrm{N}$ & $165^{\circ} 13.624^{\prime} \mathrm{W}$ & 67.18 \\
Avatanak Strait & $54^{\circ} 6.767^{\prime} \mathrm{N}$ & $165^{\circ} 28.544^{\prime} \mathrm{W}$ & 75.29 \\
Hog Neck & $41^{\circ} 43.433^{\prime} \mathrm{N}$ & $70^{\circ} 38.363^{\prime} \mathrm{W}$ & 8.53 \\
Fire Island & $61^{\circ} 10.754^{\prime} \mathrm{N}$ & $150^{\circ} 7.532^{\prime} \mathrm{W}$ & 8.44 \\
Gibson Point & $47^{\circ} 13.148^{\prime} \mathrm{N}$ & $122^{\circ} 35.320^{\prime} \mathrm{W}$ & 60.53 \\
\hline
\end{tabular}

Table 3: Location of the tidal observation sites. - Short tidal series. The rightmost column shows the approximate depth at which the measurements are made.

\begin{tabular}{|c|c|c|c|c|c|c|}
\hline \multirow[b]{2}{*}{ Location } & \multirow[b]{2}{*}{ Training Duration } & \multirow[b]{2}{*}{ Prediction } & \multicolumn{2}{|c|}{$u$} & \multicolumn{2}{|c|}{$v$} \\
\hline & & & HA & GP & HA & GP \\
\hline Derbin Strait & $12 / 06 / 10-02 / 07 / 10$ & $03 / 07 / 10-23 / 07 / 10$ & 0.3470 & 0.3433 & 0.3631 & 0.3542 \\
\hline Avatanak Strait & $11 / 06 / 10-02 / 07 / 10$ & $03 / 07 / 10-23 / 07 / 10$ & 0.2540 & 0.2442 & 0.2323 & 0.2267 \\
\hline Hog Neck & 03/06/09 - 27/06/09 & $28 / 06 / 09-21 / 07 / 09$ & 0.1873 & 0.1787 & 0.2431 & 0.2428 \\
\hline Fire Island & $13 / 07 / 02-02 / 08 / 02$ & 03/08/02 - 23/08/02 & 0.1657 & 0.1645 & 0.0895 & 0.0850 \\
\hline Gibson & $29 / 05 / 15-22 / 06 / 15$ & $23 / 06 / 15-17 / 07 / 15$ & 0.1209 & 0.1196 & 0.2081 & 0.2000 \\
\hline
\end{tabular}

Table 4: Comparison of the RMSE (in $\mathrm{m} / \mathrm{s}$ ) of the prediction using the HA and GP algorithm for short-term data analysis.

parable to that of the tidal. Such perturbations could originate from a variety of sources - internal tides [30], river discharge, meteorological forcing, estuarine currents. Prediction becomes challenging in circumstances when the low frequency non-tidal variations are considerable. To accommodate such variations, 


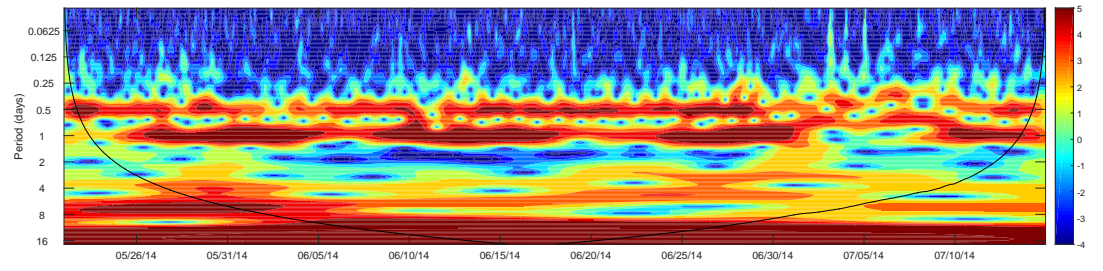

Figure 7: Wavelet transform showing the log of the wavelet power spectrum of velocity of short tidal current data series at Port of Beaumont. The analysis is performed using them methods of 28] and 29]. The Morlet wavelet has been used in the analysis and the black line encloses regions of greater than $95 \%$ confidence for a red noise process with a lag- 1 coefficient of 0.72 .

the mean of the GP can be modelled with another GP - meanGP:

$$
m \sim \mathcal{G P}\left(m_{\text {mean }}, k_{\text {mean }}\right)
$$

wavelet transform is performed on the current velocities to obtain some qualitative description of the characteristics of the tidal current data series. Wavelet 
transform [31] has been applied to a wide variety of signal processing problems [32], and unlike Fourier transform, it provides a temporal description of the frequency spectrum, which entails a loss in frequency resolution, but gain in some temporal description. Figure 7 shows the log of the wavelet power spectrum of the current velocity plotted versus the period of the tidal constituents (abscissa in $\log _{2}$ scale) and time (ordinate). As it can be noticed, the current data possess significant amount of non-tidal energy especially at low frequencies. It is not possible to capture such variations, appropriately, using the HA approach. Note, the wavelet transform can resolve different tidal species (diurnal, semi-diurunal) band, but not the tidal constituents within the species [33]. Figure 9 shows the current data at the location from 22/May/2014 to 14/July/2014 - which is 54 days of data, and predictions are made over the last six days (shown in blue in 240 the figure), half-a-day in advance. For this particular case, twenty four hours moving average of the tidal current velocities are computed and the meanGP is employed on the data points. Table 5 shows the RMSE of the half-a-day ahead predictions. Significant reductions in error have been achieved with an overall (considering 7 days) decrease of 43 percent in the $u$ velocity and 40 percent in the $v$ velocity. This demonstrates that even in the absence of additional information, ML can be used to enhance current predictions.

\section{Discussion}

The paper presents an alternate approach to the prediction of tidal currents, different from all known existing methods. Comparisons are made with the HA for some real tidal current datasets (see tables 2 and 4) which are almost stationary (HA performs good), and the GP approach is shown to outperform the HA. The advantages of the machine learning approach lies in its flexibility and applicability in modelling a wide variety of real world situations. In the GP framework, structures in the data are captured primarily through kernel 

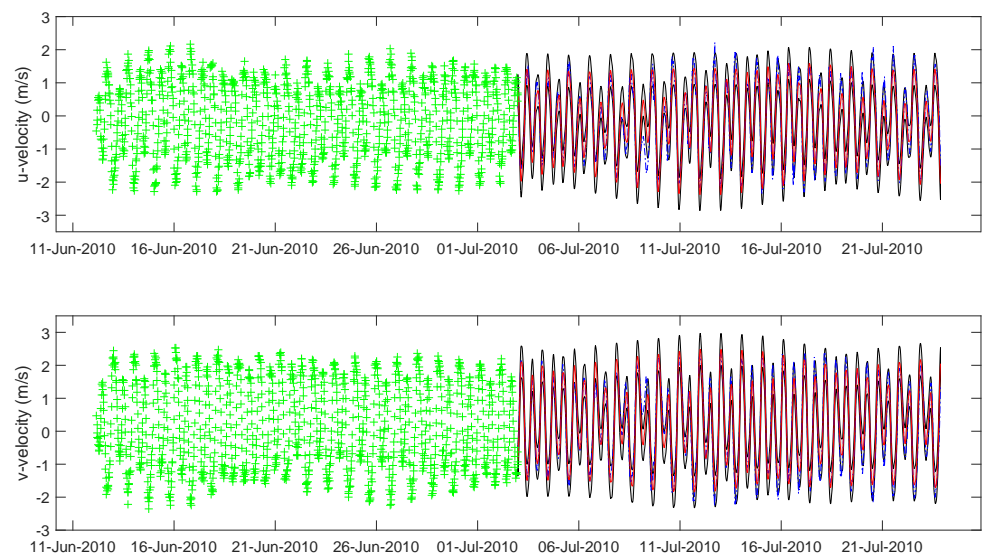

Figure 8: Training and prediction of a short tidal current data series at Gibson Point.
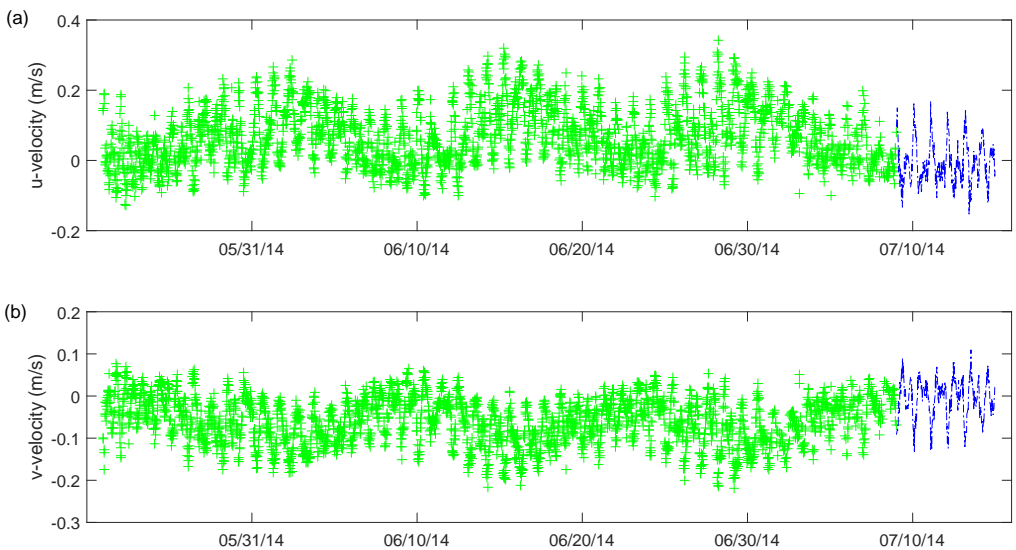

Figure 9: Variation of horizontal current velocities (a) $u$ and (b) $v$ at Port of Beaumont (USA) between 22/May/2014 to 14/July/2014. The points in green indicate the initial training data while the blue dashed-dotted line indicate the data points at the test locations which we intend to predict half-a-day in advance.

functions which comprises of hyperparameters, and they are typically learned from the data. Unlike in HA which strictly requires the frequencies of the constituents to be predetermined, the periodicity hyperparameter in the GP do 


\begin{tabular}{|c|c|c|c|c|c|}
\hline \multirow[b]{2}{*}{ Prediction } & \multirow[b]{2}{*}{ Duration } & \multicolumn{2}{|c|}{$u$} & \multicolumn{2}{|c|}{$v$} \\
\hline & & $\mathrm{HA}$ & GP & $\mathrm{HA}$ & GP \\
\hline \multirow[t]{2}{*}{ 09/07/14 } & 1st half & 0.0693 & 0.0531 & 0.0444 & 0.0385 \\
\hline & 2nd half & 0.0512 & 0.0390 & 0.0346 & 0.0287 \\
\hline \multirow[t]{2}{*}{$10 / 07 / 14$} & 1st half & 0.0456 & 0.0349 & 0.0346 & 0.0257 \\
\hline & 2nd half & 0.0771 & 0.0575 & 0.0496 & 0.0409 \\
\hline \multirow[t]{2}{*}{$11 / 07 / 14$} & 1st half & 0.0679 & 0.0490 & 0.0380 & 0.0289 \\
\hline & 2nd half & 0.0819 & 0.0561 & 0.0534 & 0.0410 \\
\hline \multirow[t]{2}{*}{$12 / 07 / 14$} & 1st half & 0.1068 & 0.0578 & 0.0632 & 0.0397 \\
\hline & 2nd half & 0.0890 & 0.0520 & 0.0564 & 0.0289 \\
\hline \multirow[t]{2}{*}{$13 / 07 / 14$} & 1st half & 0.1172 & 0.0447 & 0.0693 & 0.0254 \\
\hline & 2nd half & 0.0945 & 0.0408 & 0.0619 & 0.0232 \\
\hline \multirow[t]{2}{*}{$14 / 07 / 14$} & 1st half & 0.1128 & 0.0378 & 0.0712 & 0.0255 \\
\hline & 2nd half & 0.0987 & 0.0361 & 0.0577 & 0.0195 \\
\hline
\end{tabular}

Table 5: RMSE of the half-a-day ahead predictions at Port of Beaumont. 1st half indicates the duration between midnight and noon and 2nd half indicates between noon till midnight on the same day. The interval between consecutive data readings is 24 mins, although there do exist some minor gaps in the data. not necessarily need to be known beforehand.

Additional information can be incorporated into the GP model which can enable it to make better short-term predictions e.g. meteorological forcing parameters like wind speed and direction which are known to influence the tidal current variations can be considered as extra inputs to the GP model. One can also be interested in knowing the tidal current variations at an unknown loca- 

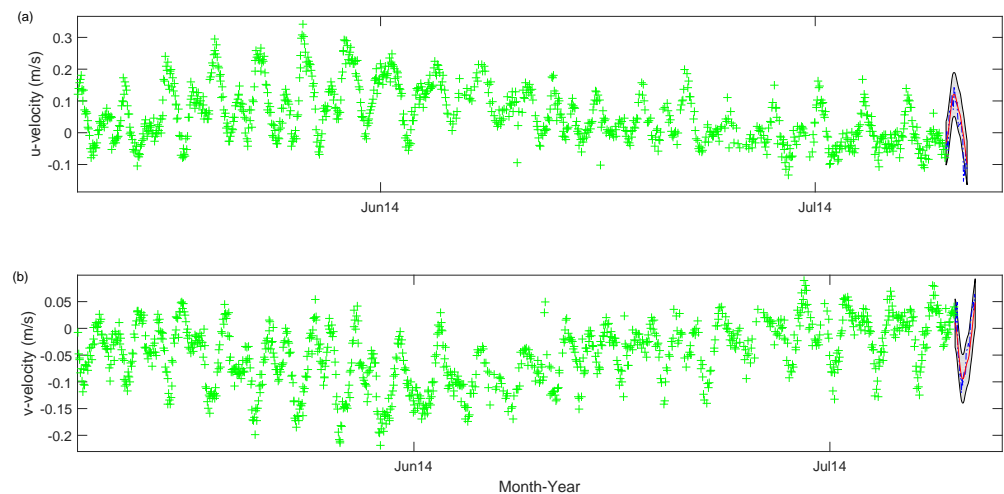

Figure 10: Current velocity prediction half a day in advance. Note, the figure doesn't show the entire training data (in green) used for the predictions

tion given the data of some nearby locations. In fact, vessel mounted acoustic Doppler current profilers (ADCPs) are often used to gather data over a spatial domain within a certain timeframe. It is not possible to perform robust predictions using classical techniques for such datasets. Such cases would involve modelling the spatial variability in addition, and a GP model with an appropriate kernel function can be used for such purpose. With the problem being formulated in the machine learning framework, we have at our disposal a wide range of possibilities in modelling tidal datasets. Methods or algorithms can be chosen depending on the specifics of the dataset. Some other features of the presented methodology are:

- Non-stationarity could be introduced by some non-linear transformation (warping) of the inputs [see e.g. 34] or outputs [see e.g. 35], and then using the normal kernel functions (stationary) on the mapped space.

- There can be issues in modelling tidal current time series using HA when the size of the basis matrix becomes large. Sparse algorithms of the GP - sparse spectrum GP [36], fully independent training conditional (FITC) 37] can be used in such circumstances. The algorithms have been shown to handle large datasets successfully. 
- The likelihood function in GP models the noise in the data. Although the other noise models - Laplace, Warped Gaussian can be considered within the same framework. Exact inference is not possible in such cases and approximate deterministic inference techniques (Variational Bayes, Expectation Propagation, Laplace)[see 38] or numerical sampling techniques (like MCMC) [39] could be applied.

- The focus of the present paper is on tidal currents, however the method is easily applicable to the prediction of tidal heights. The latter involves just 1-D output (tidal heights) and hence is more straightforward.

- Effect of additional unknown frequencies in the tidal signal data could be incorporated by considering additional periodic kernel functions with 'free' periodicity hyperparameter.

The GP formulation is a non-parametric approach, and therefore has added flexibilities which are not provided by parametric methods like the HA. The periodic kernel function chooses from the space of harmonic functions and the output may not necessarily be exactly sinusoidal.

\section{Conclusion}

The problem of tidal current prediction is formulated in the framework of Bayesian GP. The goal of this work was develop robust prediction method which can address some of the shortcoming of the traditional approaches. The kernel function of the GP captures the structures in the data and amongst the wide choice of kernel functions, the periodic kernel function is in particular relevant for the present application due to its harmonic nature, without being restricted to just sinusoidal variations. The hyperparameters of the kernel functions are inferred either deterministically using MAP approach or stochastically using MCMC, with the former method being preferred for large datasets. The 
probabilistic nature of the machine learning approach enables it to handle uncertainty and noise, and the method generates confidence intervals directly in the time-domain (unlike HA), which would be useful in practical applications. The machine learning approach is also shown to capture non-harmonic variation using the exponentiated quadratic kernel function and can make better predictions in the short-term. We analyze and perform predictions on several sets of field data at various locations around USA. In general, the GP framework provide additional flexibilities not provided by the traditional techniques and can enhance tidal analysis. We have demonstrated that the GP based approach can achieve better accuracy than the traditional HA over a wide range of cases and varied signal characteristics. In general, enhancing prediction of currents requires better understanding of the physical processes such as the conversion of barotropic to baroclinic tides, decay of internal tides into turbulence [40], meteorological effects, etc. Availability of data related to the variation of relevant parameters e.g. the meteorological forcing variables (wind stress intensity and direction) can aid in the construction of better predictive models and exploit the full potential of the machine learning algorithms.

\section{Acknowledgements}

This work is supported by the EPSRC, UK (Grant Number: EP/M021394/1). The authors are grateful to National Oceanic and Atmospheric Administration, USA for the availability of current data. The authors also thank the developers of the GPML and UTide code.

[1] R. Pawlowicz, B. Beardsley, S. Lentz, Classical tidal harmonic analysis including error estimates in matlab using t_tide, Computers \& Geosciences 28 (8) (2002) 929-937.

[2] M. Foreman, J. Cherniawsky, V. Ballantyne, Versatile harmonic tidal analysis: Improvements and applications, Journal of Atmospheric and Oceanic technology 26 (4) (2009) 806-817. 
[3] K. E. Leffler, D. A. Jay, Enhancing tidal harmonic analysis: Robust (hybrid 11/12) solutions, Continental Shelf Research 29 (1) (2009) 78-88.

[4] W. H. Munk, D. E. Cartwright, Tidal spectroscopy and prediction, Philosophical Transactions of the Royal Society of London A: Mathematical, Physical and Engineering Sciences 259 (1105) (1966) 533-581.

[5] D. E. Cartwright, A unified analysis of tides and surges round north and east britain, Philosophical Transactions of the Royal Society of London A: Mathematical, Physical and Engineering Sciences 263 (1134) (1968) 1-55.

[6] D. A. Jay, E. P. Flinchem, A comparison of methods for analysis of tidal records containing multi-scale non-tidal background energy, Continental Shelf Research 19 (13) (1999) 1695-1732.

[7] C. T. Friedrichs, D. G. Aubrey, Non-linear tidal distortion in shallow wellmixed estuaries: a synthesis, Estuarine, Coastal and Shelf Science 27 (5) (1988) 521-545.

[8] W. R. Geyer, Three-dimensional tidal flow around headlands, Journal of Geophysical Research: Oceans 98 (C1) (1993) 955-966.

[9] S. Roberts, M. Osborne, M. Ebden, S. Reece, N. Gibson, S. Aigrain, Gaussian processes for time-series modelling, Philosophical Transactions of the Royal Society of London A: Mathematical, Physical and Engineering Sciences 371 (1984) (2013) 20110550.

[10] Z. Ghahramani, Probabilistic machine learning and artificial intelligence, Nature 521 (7553) (2015) 452-459.

[11] G. Matheron, The intrinsic random functions and their applications, Advances in applied probability (1973) 439-468.

[12] P. D. Thompson, Optimum smoothing of two-dimensional fields, Tellus 8 (1956) 384-393. 
[13] C.-J. Kim, C. R. Nelson, Has the us economy become more stable? a bayesian approach based on a markov-switching model of the business cycle, The review of Economics and Statistics 81 (4) (1999) 608-616.

[14] B. D. Ripley, Spatial statistics, Vol. 575, John Wiley \& Sons, 2005.

[15] C. E. Rasmussen, C. K. Williams, Gaussian processes for machine learning, the MIT Press.

[16] D. Sarkar, M. Osborne, T. Adcock, A machine learning approach to the prediction of tidal currents, in: The Proceedings of the 26th (2016) International Ocean and Polar Engineering Conference, Rhodes, Greece, 2016, pp. $692-700$.

[17] M. Foreman, Manual for tidal currents analysis and prediction.

[18] M. Foreman, Manual of tidal heights analysis and prediction., Pacific Marine sciences Report 77-10, Institute of Ocean Sciences, Patricia Bay.

[19] G. Godin, The analysis of tides., University of Toronto Press.

380 [20] D. MacKay, Information theory, inference and learning algorithms, Cambridge University Press, 2003.

[21] C. E. Rasmussen, Evaluation of Gaussian processes and other methods for non-linear regression, Ph.D. thesis, Citeseer (1996).

[22] M. A. Alvarez, N. D. Lawrence, Computationally efficient convolved multiple output Gaussian processes, The Journal of Machine Learning Research 12 (2011) 1459-1500.

[23] J. C. Pinheiro, D. M. Bates, Unconstrained parametrizations for variancecovariance matrices, Statistics and Computing 6 (3) (1996) 289-296.

[24] M. A. Osborne, S. J. Roberts, A. Rogers, N. R. Jennings, Real-time information processing of environmental sensor network data using bayesian Gaussian processes, ACM Transactions on Sensor Networks (TOSN) 9 (1) (2012) 1. 
[25] D. Codiga, Unified tidal analysis and prediction using the utide matlab functions. technical report 2011-01. graduate school of oceanography, university of rhode island, narragansett, ri. 59pp, ftp://www.po.gso.uri.edu/pub/downloads/codiga/pubs/2011CodigaUTide-Report.pdf.

[26] J. Hensman, N. Fusi, N. D. Lawrence, Gaussian processes for big data, in: Conference on Uncertainty in Artificial Intelligence, 2013, pp. 282-290. URL http://arxiv . org/abs/1309.6835

[27] Y. Ding, R. Kondor, J. Eskreis-Winkler, Multiresolution Kernel Approximation for Gaussian Process Regression, arXiv:1708.02183 [stat]. URL http://arxiv.org/abs/1708.02183

[28] C. Torrence, G. P. Compo, A practical guide to wavelet analysis, Bulletin of the American Meteorological society 79 (1) (1998) 61-78.

[29] P. C. Liu, G. S. Miller, Wavelet transforms and ocean current data analysis, Journal of atmospheric and oceanic technology 13 (5) (1996) 1090-1099.

[30] C. Wunsch, Internal tides in the ocean, Reviews of Geophysics 13 (1) (1975) $167-182$.

[31] I. Daubechies, et al., Ten lectures on wavelets, Vol. 61, SIAM, 1992.

[32] S. Mallat, A wavelet tour of signal processing, Academic press, 1999.

[33] L. Guo, M. van der Wegen, D. A. Jay, P. Matte, Z. B. Wang, D. Roelvink, Q. He, River-tide dynamics: Exploration of nonstationary and nonlinear tidal behavior in the yangtze river estuary, Journal of Geophysical Research: Oceans 120 (5) (2015) 3499-3521.

[34] P. D. Sampson, P. Guttorp, Nonparametric estimation of nonstationary spatial covariance structure, Journal of the American Statistical Association 87 (417) (1992) 108-119. 
[35] E. Snelson, C. E. Rasmussen, Z. Ghahramani, Warped Gaussian processes, Advances in neural information processing systems 16 (2004) 337-344.

[36] M. Lázaro-Gredilla, J. Quiñonero-Candela, C. E. Rasmussen, A. R. Figueiras-Vidal, Sparse spectrum Gaussian process regression, The Journal of Machine Learning Research 11 (2010) 1865-1881.

[37] J. Quinonero-Candela, C. E. Rasmussen, A unifying view of sparse approximate Gaussian process regression, The Journal of Machine Learning Research 6 (2005) 1939-1959.

[38] T. P. Minka, Expectation propagation for approximate bayesian inference, in: Proceedings of the Seventeenth conference on Uncertainty in artificial intelligence, 2001, pp. 362-369.

[39] C. Bishop, Pattern recognition and machine learning, Springer, 2006.

[40] G. Egbert, R. Ray, Significant dissipation of tidal energy in the deep ocean inferred from satellite altimeter data, Nature 405 (6788) (2000) 775-778. 